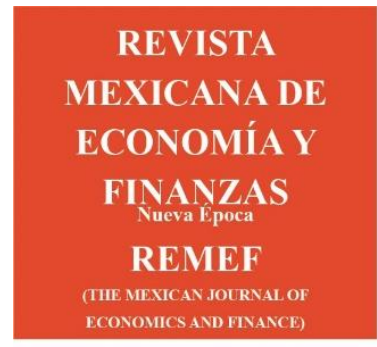

Revista Mexicana de Economía y Finanzas, Nueva Época

Volumen 16 Número 4, Octubre - Diciembre 2021, pp. 1-22, e505

DOI: https://doi.org/10.21919/remef.v16i4.505

(Recibido: 25/mayo/2020, aceptado: 22/octubre/2020, publicado: 5/mayo/2021)

\title{
Autómata Evolutivo (AE) para el mercado accionario usando martingalas y un algoritmo genético
}

\author{
Jaime Alberto Gómez Vilchis ${ }^{1}$ - Universidad Nacional Autónoma de México, México \\ Federico Hernández Álvarez - Universidad Nacional Autónoma de México, México \\ Luis Ignacio Román de la Sancha - Universidad Nacional Autónoma de México, México
}

El objetivo de este trabajo es desarrollar un Autómata Evolutivo (AE) que opera con base a un modelo de martingalas con el que se definen estrategias de inversión, las cuales utilizan información inmediata histórica, límites de ganancia, pérdida y tiempos de permanencia; brinda señales de compra, venta o mantener la posición del activo basadas en la combinación óptima de medias móviles seleccionadas mediante un algoritmo genético. Se probó para dos índices accionarios antes, durante y después de la crisis subprime, mostrando que, cuando los mercados estaban en fase alcista, la estrategia comprar-manter $(\mathrm{BH})$ generó un rendimiento superior al $A E$; en contraste, para el periodo de crisis observado, el $A E$ logró un rendimiento mayor; finalmente, en todo el periodo de prueba, el rendimiento del $A E$ fue superior. El $A E$ tiene la restricción que solo puede ser usado por una acción/índice por periodo, aunque esto puede ser solventado al ciclarlo por el número de instrumentos del portafolio. La autenticidad del trabajo radica en la combinación de modelos que se complementan para generar un sistema que ayuda en la toma de decisiones.

Clasificación JEL: C61, C63, G12, G17, G14.

Palabras clave: Autómata evolutivo, martingalas, tiempos de paro, medias móviles, algoritmo genético.

\section{Evolutionary Automaton (AE) for the Stock Market Using Martingales and a Genetic Algorithm}

The aim of this paper is to develop an Evolutionary Automata (EA) that operates considering a martingale model, which defines investment strategies using immediate historical information, profit or lost limits and stopping time. The EA offers signals to buy, sell or hold a determinate stock, these signals are based on the optimal combination of simple moving average through a genetic algorithm. The EA was tested with two stock indices, before, during and after the subprime crisis. Finding that when the stock markets were in bull market, the Buy-Hold (BH) strategy presented superior performance than the EA; in contrast, during the crisis period, the performance of the EA was better than $\mathrm{BH}$, finally, for all of the test period, the performance of the EA was superior. The EA have the restriction that can be used by one stock/index for period, though this can be solved by cycling the number of instruments of the portfolio. The authenticity of this research is the combination of the above models that match between them in order to generate a system that helps investor decisions. JEL Classification: C61, C63, G12, G17, G14.

Keywords: Evolutionary Automata, martingales, stopping time, moving average, genetic algorithm.

${ }_{1}^{1}$ Autor de correspondencia. Departamento de Ingeniería de Sistemas, Facultad de Ciencias, UNAM

Av. Universidad № 3000, Universidad Nacional Autónoma de México, C.U., Ciudad de México, C.P. 04510, teléfono: 55-2522-3398, e-mail: jaimberto@hotmail.com

*Sin fuente de financiamiento para el desarrollo de la investigación 


\section{Introducción}

El objetivo del presente trabajo es desarrollar un Autómata Evolutivo (AE), con base a una combinación novedosa de un conjunto de conceptos probados en la literatura como mecanismos de investigación acerca de problemas relacionados con el comportamiento caótico de las cotizaciones de activos e índices en los mercados financieros. Se propone hacer uso de la modelación con martingalas, algoritmos genéticos y técnicas de análisis técnico en un sistema que los acople, para estudiar el comportamiento de los mercados financieros y probar como un autómata computacional puede utilizar estos modelos para maximizar las ganancias de los inversores y aplicarlo para la toma de decisiones de inversión. Para evaluar su desempeño, se compara el rendimiento generado por el $A E$ contra el rendimiento obtenido al usar la estrategia de comprar y mantener BH (por sus siglas en inglés, Buy and Hold) en el periodo de prueba, que abarca antes, durante y después de la crisis financiera subprime del 2007 surgida en Estados Unidos. Para evaluar el funcionamiento del $A E$ en dicho periodo, se tomaron los valores del Índice de Precios y Cotizaciones (IPC) de la Bolsa Mexicana de Valores (BMV) y el Índice Dow Jones Industrial Average (DJ) de los Estados Unidos. Se escoge este periodo por la variación y cambios significativos en las trayectorias que se presentaron, de manera que pueda extrapolarse para cualquier otro periodo futuro. Se prueba que el autómata genera rendimientos superiores a la estrategia simple BH durante un periodo que presentó fases de estabilidad, crisis y recuperación.

El autómata acopla tres conceptos utilizados en el estudio de los mercados financieros (martingalas, análisis técnico y algoritmos genéticos), utilizados en finanzas con fines de pronóstico o para realizar operaciones de compra-venta. Recientemente los autómatas se han construido con base a diferentes técnicas de inteligencia artificial y machine learning, por ejemplo: Necchi (2016), construyó un autómata que realiza operaciones de trading usando una técnica llamada aprendizaje por refuerzo (reinforcement learning), basada en un proceso de decisión tipo Markov, ver Groenewegen (1976); otro ejemplo es el propuesto por Golub et al. (2017), que desarrolló un autómata para el mercado cambiario que usa como principal método de trading el "coastline trading", éste asemeja una estrategia tipo martingala. Una característica importante que debe de considerar un autómata de trading, es su capacidad para administrar el riesgo; Päuna (2018) usa el concepto básico de límite de pérdida en su autómata como estrategia de riesgo. El uso de autómatas es una herramienta para la toma de decisiones, éstos tienen relevancia y participación en diversas actividades de inversión; Scarpati et al. (2018) menciona que el uso de autómatas genera gran valor a las firmas de servicios financieros, no sólo en el ahorro de tiempo de proceso de información, sino en mejoras en el análisis para la toma de decisiones. Durante las últimas décadas el desempeño de los autómatas y su adaptabilidad ha sido muy valorada al grado que su popularidad ha crecido, de acuerdo con Chiou (2019), entre el ochenta y noventa por ciento de las transacciones en los mercados financieros de Wall Street son operados por autómatas los cuales son recalibrados y mejorados continuamente. Los métodos numéricos con los que trabajan los autómatas son parte importante de sus estrategias optimizando su funcionamiento; en particular, uno de los métodos más usados para los autómatas de trading es el análisis técnico, sin embargo, estos tienen que ser complementados con otros métodos para volverlos más robustos y eficientes, de acuerdo con Huang et al. (2019). 
El modelo propuesto en este trabajo, y el cual es utilizado por el $A E$, propone como primer elemento las martingalas como base matemática, con estas se pueden definir estrategias para las posiciones de compra-venta. La teoría surgió con el objetivo de modelar el dinero proveniente de apuestas en juegos de azar. Este tipo de modelo representa la evolución del capital de un jugador que realiza una sucesión de apuestas justas, Rincón (2012). Esta teoría se ha relacionado con hipótesis financieras y ha sido una de las principales herramientas en el estudio de los procesos estocásticos. En un inicio Fama y Blume (1966), relacionaron las martingalas con la utilidad esperada del inversionista. Tiempo después, Edwards et al. (2018), utilizando martingalas, probaron que la información histórica de un activo muestra indicios sobre su comportamiento futuro. Para el caso del mercado mexicano, de la Uz (2002), prueba estadísticamente que los índices IPC e INMEX para el periodo de 1993-2000 cumplen con la hipótesis de martingala. El $A E$ utiliza tanto la teoría de martingalas y filtraciones con base en información inmediata anterior. El uso de martingalas en series financieras utiliza la información histórica disponible para apoyar en la toma de decisiones, sobre esto, Protter (2015) comenta que las series financieras representadas como martingala, y utilizadas vía filtraciones, acentúan el valor de datos históricos. Así también, Gourieroux y Jasiak (2019), enfatizan la importancia del tiempo de paro cuando se desea invertir en activos que no son libres de riesgo. En los últimos años, el mercado cambiario ha sido otra área de aplicación de las martingalas, Guo (2017), encuentra que para que exista una correlación entre el tipo de cambio y una metodología econométrica propuesta con un movimiento browniano, su variación cuadrática debe ser una martingala continua. Así mismo, las criptomonedas han sido estudiadas como martingalas, como en el trabajo de Schilling (2019), que encuentra que el precio de la forma más simple de Bitcoin forma una martingala la cual está ausente de especulación.

El segundo componente del modelo propuesto son el cruce de medias móviles, las cuales han sido comúnmente utilizada en el análisis técnico como estrategias de compra-venta en mercados financieros, tienen como propósito pronosticar el precio de los activos a través del estudio de estadísticos histórico de los precios y obtener ganancias en las transacciones de compra-venta. Wang et al. (2016), menciona que es común integrar modelos de medias móviles a través de algoritmos para robustecer el análisis técnico y sus estrategias en los mercados financieros. Un método de optimización que ha sido ampliamente combinado con el análisis técnico son los algoritmos genéticos, Lobato Macedo. (2020), compara diferentes retrasos de medias móviles simples y exponenciales, así como otros métodos de análisis técnico como bandas de bollinger para determinar la combinación óptima usando un algoritmo genético, aunque Lobato Macedo lo prueba en los mercados de tipo de cambio, lo que resalta la coyuntura y adaptabilidad de estos análisis con diversas técnicas para fortalecer las estrategias.

El tercer y último elemento del modelo propuesto son los Algoritmos Genéticos (AG) utilizados para buscar el cruce de medias móviles que mejor se adapte a los cambios en los precios que el activo esté experimentando y genere el mayor rendimiento. Los AG fueron desarrollados por Holland (1975), sin embargo, fue Goldberg (1989), quien encontró en ellos aplicaciones computacionales de optimización, búsqueda y "machine learning”. De acuerdo con Allen y Karjalainen (1999), desde mediados del siglo XX existe interés en aplicar los AG, que es una rama de la inteligencia artificial, con propósitos financieros y económicos. Existen trabajos donde se ha hecho uso de AG en finanzas, combinándolos con modelos de análisis técnico para encontrar reglas predictivas, tal es el caso de Jiang y Szeto (2003), que utilizan los AG como un problema de optimización para desarrollar 
reglas de compra-venta de activos de la industria tecnológica, encontrando que su estrategia basada en AG es superior a otros métodos como las caminatas aleatorias, comprar y mantener y búsqueda exhaustiva. 0 el caso de Wang et al. (2016), que hace uso de los AG en la selección óptima de medias móviles, para la compra-venta en el mercado de futuros del petróleo; Lin et al. (2017), presenta una metodología basada en minería de datos y AG para acciones del mercado de valores, y al igual que Jiang y Wang prueba que su método supera a los métodos tradicionales de comprar y mantener tanto en el pronóstico esperado como en términos de retornos anualizados de inversión. Así mismo, los AG han sido combinados con otras técnicas para robustecer sus resultados, como es el trabajo de Chung et al. (2018), quien combinan un AG con una red neuronal para resaltar las ventajas de tiempo que ofrece un AG en la predicción de indicadores para el mercado de valores; así también, Mousinho Martins y Ferreira Neves (2020), usan una técnica de reconocimiento de patrones de cuadrícula optimizándola a través de un AG, la cual detecta patrones reduciendo el riesgo y aumentando ganancias.

Por la discusión anterior, este trabajo de investigación se muestra genuino en el uso de un proceso robótico que usa martingalas para definir estrategias que van evolucionando conforme se mueven los mercados financieros, tomando en cuenta límites de administración de riesgo para minimizar las exposiciones de los riesgos a los que se está sujeto.

La hipótesis principal de este trabajo es que el uso del $A E$, ayuda a minimizar las pérdidas durante el periodo de crisis en comparación con la estrategia $\mathrm{BH}$, y en el mismo sentido a obtener mejores rendimientos durante el periodo total de la prueba. Por otra parte, para los periodos de expansión la estrategia $\mathrm{BH}$ fue mejor debido a que la tendencia alcista del periodo de prueba únicamente presentó pequeños intervalos con tendencia negativa, lo que provocó al $A E$ tomar posiciones de venta que resultaron en pérdidas de rendimiento.

El trabajo se divide en seis secciones. Posterior a la introducción, en la sección dos se describe el periodo de tiempo, así como los datos en los que se probó el $A E$. En la tercera sección la metodología usada, la cual se basa en los conceptos de martingalas, AG y análisis técnico. En la cuarta sección se relata la prueba a realizar. En la quinta sección los resultados obtenidos y por último las conclusiones y futuras aportaciones.

\section{Datos}

Para evaluar el funcionamiento del autómata se tomaron los valores del Índice de Precios y Cotizaciones (IPC) de la Bolsa Mexicana de Valores (BMV) y el Índice Dow Jones Industrial Average (DJ) de los Estados Unidos. El IPC es una muestra de las 35 acciones más bursátiles de la bolsa Mexicana de Valores (BMV 2019), por su lado el DJ refleja el comportamiento de las 30 compañías más importantes y representativas del sector industrial de los Estados Unidos de América. El $A E$ puede ser usado con cualquier emisora de manera individual, activos independientes o incluso fondos indexados y no indexados, sin embargo, se escogieron estos índices debido a su representatividad e importancia como indicadores financieros en el mercado mexicano y estadounidense. Así como, por la existencia de fondos indexados a estos índices a los que un inversionista puede tener acceso. 
El periodo de prueba se eligió de manera que abarque escenarios de crecimiento, crisis y recuperación financiera para ambos índices. El periodo que cumple con estas características es el denominado como crisis hipotecaria subprime del 2007, que va de junio de 2007 a abril de 2009 de acuerdo con Roman, Hernandez y Rodríguez (2019). Por tal motivo, se escogió como intervalo de prueba el periodo del inicio de la crisis menos dos meses, 2 de abril de 2007, al final de la crisis más diez y siete meses, 30 de septiembre de 2010, (se tomaron meses después de la crisis para observar el comportamiento del autómata en el periodo de recuperación). La prueba atraviesa por periodos de crecimiento, crisis y recuperación para ambos índices, lo que obligará al $A E$ a ser probado tanto en escenarios favorables como adversos para generar diversas posiciones.

\section{Metodología}

En esta sección se describe la metodología usada para desarrollar el $A E$ para el mercado accionario que usa martingalas y un AG. Se brinda la definición y el uso de las martingalas como el motor de una estrategia que funciona como decisor en el tiempo para entrar, salir o mantener una posición de un activo. Así mismo, se explica cómo la martingala ayuda a definir el tiempo de paro, el cual robustece la estrategia brindando límites de inversión y el tiempo máximo que se está dispuesto a invertir. Como parte de la estrategia, se aplican indicadores de análisis técnico a través de una combinación de medias móviles simples. Adicionalmente, se emplea un AG para ayudar al autómata a evolucionar con base en la búsqueda de la mejor estrategia (combinación de medias móviles) a lo largo del tiempo de operación. Se describe el AG utilizado, así también la manera en cómo modifica la estrategia del $A E$ a través del tiempo.

\subsection{Martingalas}

Sean los rendimientos de un activo financiero en el tiempo, una sucesión de variables aleatorias independientes e idénticamente distribuidas $X_{1}, X_{2}, \ldots$, donde el rendimiento acumulado al tiempo $n$ se escribe como $S_{n}=X_{1}+X_{2}+\cdots+X_{n}$. Esta sucesión es una serie discreta de puntos en el tiempo. Por otro lado, sea una filtración $\mathfrak{I}_{n}=\sigma\left\{X_{1}, \ldots, X_{n}\right\}$ una colección creciente de $\sigma$-álgebras tal que $^{2}$ $\left\{\widetilde{I}_{m}\right\} \subseteq\left\{\widetilde{I}_{n}\right\}$ cuando $m \leq n$. La filtración se interpreta como la información hasta el instante $m$ del proceso estocástico $X_{n}$.

Un proceso estocástico $\left\{M_{n}\right\}=S_{n}=X_{1}+X_{2}+\ldots+X_{n}$ es una martingala si se satisface la siguiente condición $E\left(S_{n} \mid \Im_{n-1}\right)=E\left(S_{n} \mid X_{1}, X_{2}, \ldots, X_{n-1}\right)=S_{n-1}$. Vista la definición de martingala desde una perspectiva financiera, se puede establecer que el rendimiento esperado para el tiempo futuro $n$ está dado por la historia de los rendimientos al tiempo $n-1$, por lo tanto, se tiene que $\left\{M_{n}\right\}$ es la martingala respecto del proceso $X_{n}$.

La martingala del rendimiento acumulado de un activo $\left\{M_{n}\right\}$ se puede utilizar para definir estrategias de inversión con información como: límites de ganancia, pérdida y tiempo de mantener la inversión, para esto, se necesita introducir el concepto de variables no anticipadas y transformada de martingala.

\footnotetext{
${ }^{2} \sigma\left\{X_{1}, \ldots, X_{n}\right\}$ es la $\sigma$-álgebra generada por el proceso $X_{1}, \ldots, X_{n}$. Heurísticamente es la información a la que tenemos acceso.
} 
Una variable aleatoria no anticipada $A_{n}$, es una función que depende de la información hasta el instante $n-1$, es decir, depende únicamente de la información $\mathfrak{I}_{n-1}$, formalmente, se define una variable no anticipada como una secuencia de variables aleatorias $\left\{A_{n}: 1 \leq n<\infty\right\}$ respecto de la secuencia $\mathfrak{I}_{n}$ si para todo $1 \leq n<\infty$, se tiene $A_{n} \in \mathfrak{I}_{n-1}$. Esta variable se puede utilizar para decidir si se compra, se mantiene o se vende una acción, es decir, a través de la variable no anticipada $A_{n}$ se pueden generar estrategias que dependan de la información inmediata histórica. La restricción en $A_{n}$, permite al inversionista decidir qué acción tomar acerca de la $n$ - ésima jugada (mantener, entrar o vender el activo).

Por ejemplo, sean los rendimientos de un activo financiero $X_{1}, X_{2}, \ldots, X_{n-1}$, y la definición de $A_{n}$ de la siguiente manera:

$$
A_{n}=\left\{\begin{array}{ccc}
\text { Invertir } & \text { si } & X_{n-1} \geq X_{n-2} \geq X_{n-3} \\
\text { No Invertir } & \text { si } & X_{n-1}<X_{n-2}<X_{n-3}
\end{array}\right.
$$

La variable no anticipada $A_{n}$ se define de tal forma que si el rendimiento al tiempo $n-1$ es mayor que el de $n-2$ y a su vez es mayor al de $n-3$, entonces al tiempo $n$ se tendría que invertir, en caso que suceda la condición que al tiempo $n-1$ el rendimiento sea menor que el rendimiento de $n-2$ y este sea menor que el de $n-3$, entonces a tiempo $n$ no se tendría que invertir.

Sea la variable no anticipada $\left\{A_{n}: 1 \leq n<\infty\right\}$ así también el proceso $\left\{\widetilde{M}_{n}: 0 \leq n<\infty\right\}$ a través de $\widetilde{M}_{0}=M_{0}$ y $\widetilde{M}_{n}=M_{0}+A_{1}\left(M_{1}-M_{0}\right)+A_{2}\left(M_{2}-M_{1}\right)+\cdots+A_{n}\left(M_{n}-M_{n-1}\right)$ para $n \geq 1$ es llamado la transformada de martingala de $\left\{M_{n}\right\}$ por $A_{n}$. La característica de la transformada de martingala es que se pueden construir nuevas martingalas a partir de anteriores y éstas también serán martingalas, Steele (2001). Para el caso del autómata financiero, permite construir y probar diferentes estrategias con base en la definición de la variable no anticipada $A_{n}$.

Otra variable para construir la estrategia es $\tau$, es llamada tiempo de paro para la secuencia $\left\{\mathfrak{I}_{n}\right\}$ si $\{\tau \leq n\} \in \mathfrak{I}_{n}$ para todo $0 \leq n<\infty$ donde $\tau$ toma valores en $\{0,1,2, \ldots\}$. El tiempo de paro es una variable aleatoria que detona una acción dependiendo del resultado del juego a través del tiempo. El tiempo de paro se utiliza como opción para interrumpir el juego si se define $\min \{\tau, n\}=$ $\tau \wedge n$, además, si $\left\{M_{n}\right\}$ es una martingala respecto de $\left\{\widetilde{I}_{n}\right\}$ entonces el proceso $\left\{M_{\tau \wedge n}\right\}$ también es una martingala respecto de $\left\{\mathfrak{I}_{n}\right\}$. Para la prueba véase Steele (2001).

Si se definen los límites $A$ y $B$ como límite de ganancia y pérdida respectivamente donde $A, B \in \mathbb{R}$, el tiempo de paro (tao) se escribe como $\tau=\min \left\{n: X_{n}=A\right.$ ó $\left.X_{n}=-B\right\}$, con estos límites, se dice que $\tau$ es el tiempo de paro en llegar a $A$ o - $B$ antes de $n$ tiradas, entonces se puede decir que la martingala se detendrá si el rendimiento generado a través de ésta cruza los límites $A$ o $-B$. Del hecho de que $\widetilde{M}_{n}$ sea una martingala, también se dice que $\widetilde{M}_{\tau \wedge n}$ es una martingala respecto del proceso $\left\{\mathfrak{I}_{n}\right\}$, Steele (2001).

Mediante las martingalas se pueden definir estrategias a través de variables no anticipadas las cuales mandaran alertas que informaran al inversionista acerca de la toma de decisiones. Es importante mencionar que el autómata, a través de la transformada de martingala, puede recibir $n$ estrategias que ayuden al inversor en la toma de decisiones sobre un activo financiero, sin embargo, solo se mostrará una de ellas que es la que utiliza medias móviles simples y un AG. 


\subsection{Medias móviles como variables no anticipadas y un AG}

El autómata usa como motor principal las martingalas, éstas necesitan de una variable no anticipada $A_{n}$ que funcione como estrategia y defina la posición de compra o venta para el activo financiero; para esto, se hace uso de una combinación del cruce de medias móviles simples y un AG simple.

Las medias móviles simples es uno de los más sencillos instrumentos de trading en el análisis técnico. El cruce de éstas indicará una posición de compra o venta. Sea la generación de promedios móviles, denotado por $M A_{t}$, donde $t$ indica el número de datos que serán usados para el promedio. Por ejemplo, si se tiene la siguiente desigualdad $M A_{1}>M A_{25}$ entonces se crean promedios móviles de posiciones diarias y otro de promedios móviles calculados cada 25 días; la señal de compra se dará cuando el periodo corto (el que promedia posiciones diarias) cruza por encima del promedio largo (el que está compuesto del promedio de los últimos 25 datos) y vende si el promedio diario está por debajo del promedio de 25 días, es decir, si $M A_{1}<M A_{25}$.

En general el detonador de la posición de compra o venta será el cruce de medias móviles simples que dependerán de los promedios de $t=t_{1}, t_{2}, t_{3}, t_{4}$, es decir de la tupla $M A_{t_{1}}, M A_{t_{2}}, M A_{t_{3}}, M A_{t_{4}}$, como lo marca Jiang y Szeto (2003). Por ejemplo, si se cumple la siguiente desigualdad $M A_{t_{1}}>M A_{t_{2}}>M A_{t_{3}}>M A_{t_{4}}$, entonces se manda una señal de compra; en caso de que se cumpla la desigualdad $M A_{t_{1}}<M A_{t_{2}}<M A_{t_{3}}<M A_{t_{4}}$ se manda una señal de venta. Es importante mencionar que existen otras combinaciones de medias móviles que pudieran dar mejores resultados al inversor, sin embargo, para encontrar la mejor combinación de medias móviles es necesario hacer uso de una herramienta de optimización para encontrarla la combinación de forma eficiente. La variable no anticipada $A_{n}$ del proceso $\widetilde{M}_{\tau \wedge n}$ queda definida de la siguiente forma:

$$
A_{n}=\left\{\begin{array}{lll}
\text { Compra/Invertir } & \text { si } & M A_{t_{1}}>M A_{t_{2}}>M A_{t_{3}}>M A_{t_{4}} \\
\text { Venta/No Invertir } & \text { si } & M A_{t_{1}}<M A_{t_{2}}<M A_{t_{3}}<M A_{t_{4}}
\end{array}\right.
$$

Como se visualiza en la variable no anticipada, una estrategia se define con una combinación particular de la tupla $\left\{M A_{t_{1}}, M A_{t_{2}}, M A_{t_{3}}, M A_{t_{4}}\right\}$ de compra y otra de venta. La idea general es que para cada instante $n$, las posiciones (combinación) de las medias móviles cambien de acuerdo a las condiciones de mercado del activo al que se está haciendo trading en $n-1$, de forma que el cruce óptimo de medias móviles defina la estrategia al tiempo siguiente. Para encontrar la tupla óptima se utiliza un AG simple que tiene como misión localizar la combinación de medias móviles que generen el mayor rendimiento en el periodo de prueba, lo que ahorra tiempo en comparación con evaluar las tuplas de compra y venta de forma secuencial.

\subsection{Algoritmos genéticos simples}

Los AG son herramientas de búsqueda basadas en la selección natural y la genética que combinan la supervivencia a través de una función objetivo. Una población de individuos creada de forma artificial en estos algoritmos, compite entre sí y se adapta tomando pedazos de historia a través de una función objetivo y de operadores genéticos. Un AG simple se compone de tres operadores genéticos: Reproducción, Cruce y Mutación, Goldberg (1989), y llega a su cometido a través del copiado e 
intercambio parcial de estructuras de cadenas de información. Cada individuo ${ }^{3}$ representa una solución al problema y están hechos a base de estructuras de cadenas de información. Un cruce de medias móviles interpreta una posible solución que se compone de una combinación de la tupla $\left\{M A_{t_{1}}, M A_{t_{2}}, M A_{t_{3}}, M A_{t_{4}}\right\}$ de compra o inversión si $M A_{t_{1}}>M A_{t_{2}}>M A_{t_{3}}>M A_{t_{4}}$ y una combinación de venta o no inversión si $M A_{t_{1}}<M A_{t_{2}}<M A_{t_{3}}<M A_{t_{4}}$, esto se puede representar de la siguiente manera:

\begin{tabular}{ccccc}
\multicolumn{5}{c}{$M A_{t_{1}}, M A_{t_{2}}, M A_{t_{3}}, M A_{t_{4}}$} \\
Compra & $\downarrow$ & $\downarrow$ & $\downarrow$ & $\downarrow$ \\
& 1 & 2 & 3 & 4
\end{tabular}

\begin{tabular}{ccccc}
\multicolumn{4}{c}{$M A_{t_{4}}, M A_{t_{3}}, M A_{t_{2}}, M A_{t_{1}}$} \\
Venta & $\downarrow$ & $\downarrow$ & $\downarrow$ & $\downarrow$ \\
& 4 & 3 & 2 & 1
\end{tabular}

El individuo queda descrito mediante las cadenas $(1,2,3,4)$ y $(4,3,2,1)$, para el caso del ejemplo anterior. El AG se encargará de ir variando las cadenas para encontrar al individuo con el mayor rendimiento producido en la función objetivo, la función objetivo es el rendimiento acumulado $S_{n-1}$ producido por evaluar las tuplas en el periodo de prueba del AG.

Se propone los siguientes pasos para que el AG simple llegue a una solución óptima en $n$ simulaciones:

1. Definición de la población inicial y el número de simulaciones.

2. Generación de la población de forma aleatoria.

3. Evaluación de cada individuo de la población por la función objetivo.

4. Selección de los individuos más aptos mediante el operador reproducción.

5. Generación de descendientes a través del operador cruce.

6. Aplicación del operador mutación.

7. En caso de que el número de individuos sea menor al número de individuos por población se crean nuevos individuos diferentes a los anteriores de forma aleatoria.

8. Repetir los pasos 3 a 6 para los nuevos individuos hasta completar el número de simulaciones.

9. Obtener el óptimo de los individuos.

Antes de empezar a mencionar la estructura del AG, así como la función de los operadores en el cruce de medias móviles, se define la función objetivo que calificará las combinaciones de medias móviles. Para este caso, la función objetivo se define como el rendimiento acumulado $S_{n-1}$, generado mediante la evaluación diaria del cruce de medias móviles en un intervalo de tiempo de $n-m$ a $n-$ 1 donde $m<n$. Por ejemplo, para el individuo $\left\{M A_{t_{1}}, M A_{t_{2}}, M A_{t_{3}}, M A_{t_{4}}\right\},\left\{M A_{t_{4}}, M A_{t_{3}}, M A_{t_{2}}, M A_{t_{1}}\right\}$ la variable no anticipada será:

$$
A_{n}=\left\{\begin{array}{lll}
\text { Compra } / \text { Invertir } & \text { si } & M A_{t_{1}}>M A_{t_{2}}>M A_{t_{3}}>M A_{t_{4}} \\
\text { Venta } / \text { No Invertir } & \text { si } & M A_{t_{1}}<M A_{t_{2}}<M A_{t_{3}}<M A_{t_{4}}
\end{array}\right.
$$

\footnotetext{
${ }^{3}$ En la literatura, los individuos de una población para un AG también son llamados cromosomas.
} 
La variable no anticipada evaluada en la función objetivo genera $S_{n-1}$, donde $S_{n-1}$ representa el rendimiento acumulado obtenido por la aplicación de las reglas de cruce de medias móviles en el intervalo de tiempo de $n-m, \ldots, n-1$.

El rendimiento acumulado obtenido es el resultado de la función objetivo y se utiliza para identificar a los individuos más aptos. Siguiendo el ejemplo anterior, sean los siguientes individuos evaluados mediante la función objetivo en el intervalo $n-m, \ldots, n-1$.

$$
\begin{array}{cl}
S_{n-1}=10 \% & \text { Ind } 1=(1,2,3,4)(4,3,2,1) \\
S_{n-1}=0.05 \% & \text { Ind } 2=(1,2,4,3)(3,4,2,1) \\
S_{n-1}=3 \% & \text { Ind } 3=(1,4,3,2)(2,3,4,1)
\end{array}
$$

Como resultado de la función objetivo, cada individuo obtuvo un rendimiento acumulado, el cual será usado en el operador reproducción.

Una población es un conjunto de individuos o cromosomas que forman parte de la solución al problema, para este caso, es un conjunto de duplas de compra y venta. Los individuos se crean de forma aleatoria, es decir, la posición de las medias móviles de compra y venta se determinan de manera aleatoria. El número de simulaciones representan las generaciones por las que se transforma la población, es decir, para cada generación, los individuos son evaluados por la función objetivo, en donde, aquellos que tengan mayor calificación serán los que tengan mayor probabilidad de reproducirse. Cada generación de individuos pasa por los operadores reproducción, cruce y mutación.

La reproducción es el proceso en el cual la información de un individuo es copiada de acuerdo con el rendimiento de su función objetivo, es decir, aquellos individuos que tendrán derecho a reproducirse serán los que sean más aptos. La elección de individuos se hace de forma aleatoria mediante la ruleta sesgada ${ }^{4}$ que ejemplifica Goldberg (1989). Es decir, cada individuo participa en la elección de ser escogido, pero no todos los individuos tienen la misma probabilidad. La elección de individuos se hará de un muestreo con reemplazo donde la probabilidad de ser escogido dependerá de su desempeño en la función objetivo. Aquellos individuos que hayan tenido los mayores rendimientos tendrán mayor oportunidad de reproducirse. Por ejemplo, de los individuos que pasaron por la función objetivo, se obtienen las siguientes probabilidades:

Tabla 1. Probabilidades

\begin{tabular}{|l|l|l|}
\hline Individuo & $\begin{array}{l}\text { Rendimiento } \\
\text { acumulado }\end{array}$ & $\begin{array}{l}\text { Probabilidad de } \\
\text { reproducción }\end{array}$ \\
\hline Individuo 1 & $10 \%$ & $76.6 \%$ \\
\hline Individuo 2 & $0.05 \%$ & $0.4 \%$ \\
\hline Individuo 3 & $3 \%$ & $23.0 \%$ \\
\hline Total & $13 \%$ & $100 \%$ \\
\hline
\end{tabular}

Fuente: Elaboración propia

\footnotetext{
${ }^{4}$ Las casillas tienen distintas probabilidades, no son del mismo tamaño.
} 
La probabilidad del individuo 1 de ser escogido es del 76.6\%, para el individuo 2 del $0.4 \%$ y para el individuo 3 del 23\%, estas probabilidades se obtienen como la proporción del rendimiento acumulado por individuo respecto del total; en este ejemplo el individuo 1 y 3 tendrán mayor probabilidad de ser escogidos para reproducirse, de acuerdo con el desempeño mostrado.

El cruce se refiere al intercambio de información a través del apareamiento aleatorio, del cual, nuevos individuos surgen con información genética de los padres. Los padres que sean escogidos para reproducirse cruzarán información que les heredarán a sus descendientes. El cruce de información se hace mediante el "Partial Mapped Crossover" (PMX) que establecen Jiang y Szeto (2003). El PMX se realiza de forma independientemente tanto en las posiciones de compra como en las posiciones de venta. Se escogen de forma aleatoria dos valores enteros $P_{1}, P_{2}=1,2, \ldots, k$, que denotan el número de medias móviles por posición de compra o venta, donde $P_{1}<P_{2}$ y $k=$ \#Medias Móviles por Posición, para este trabajo $k=4$. Se cambia información entre individuos de la posición respectiva a $P_{1} y P_{2}$, tomando en cuenta que no puede haber valores repetidos entre medias móviles. De cada padre saldrá un descendiente que tendrá información "genética" de sus sucesores.

De acuerdo con el ejemplo, sean los individuos 1 y 3 escogidos para cruzar información; exclusivamente para las posiciones de compra tenemos las siguientes cadenas $(1,2,3,4)$ y $(1,4,3,2)$. Se obtienen las posiciones $P_{1}=2$ y $P_{2}=3$ generadas de forma aleatoria. La posición 2 de la cadena de compra del individuo 1 será intercambiada por la posición 3 de la cadena de compra del individuo 2 , cuidando que no existan valores repetidos. Una vez aplicado el operador cruce se crean dos descendientes que tendrían la estructura $(1,3,2,4)$ y $(1,4,2,3)$ respectivamente para las cadenas de compra. Para las posiciones de venta se hace el mismo procedimiento. Para mayor explicación ver Jiang y Szeto (2003).

La mutación, así como en la selección natural, se da en proporciones muy pequeñas, esta modifica la información del individuo. Todos los individuos están sujetos a sufrir una mutación, sin embargo, la tasa de mutación es baja, para este trabajo corresponde al 0.001, de acuerdo con Goldberg (1989). La mutación consiste en el intercambio de dos puntos propuesto por Jiang y Szeto (2003), el cual consiste en escoger dos valores enteros $P_{1}$ y $P_{2}$ con $P_{1}, P_{2}=1,2, \ldots, k$, donde $P_{1}<P_{2}$ y $k=\#$ Medias Móviles por posición, similares al procedimiento PMX que se establece en el operador cruce. Con la diferencia que no se da un intercambio entre individuos, se cambian los valores correspondientes a las posiciones $P_{1}$ y $P_{2}$. Este intercambio se genera de forma independiente tanto para la parte de compra como para la parte de venta. El ciclo se repite para los individuos que no han sido calificados. En caso de que el número de descendientes sea menor al número de individuos por generación, se generan de forma aleatoria nuevos individuos diferentes a los existentes.

La estrategia óptima será aquella que genere el rendimiento más alto obtenido por el AG simple en el periodo de prueba. Por ejemplo, sea las combinaciones óptimas para la corrida inicial del ejercicio de prueba las siguientes ${ }^{5}$ :

$$
\left\{M A_{1}, M A_{5}, M A_{10}, M A_{25}\right\},\left\{M A_{25}, M A_{1}, M A_{10}, M A_{5}\right\} \text { Para el IPC. }
$$

\footnotetext{
${ }^{5}$ El $A E$ puede ser usado para cualquier acción, en la sección 4 se explica el uso y el porqué de estas series.
} 
$\left\{M A_{1}, M A_{5}, M A_{10}, M A_{25}\right\},\left\{M A_{1}, M A_{10}, M A_{5}, M A_{25}\right\}$ Para el DJ.

Los conceptos mencionados anteriormente fueron codificados en un programa computacional el cual, recolecta datos diarios del precio de una acción y ejecuta la martingala de manera que cada vez que se extrae nuevas cotizaciones del activo se decide sobre la postura de inversión que sea más conveniente.

\section{Prueba}

La prueba ocurre en intervalos de tiempo o rondas: el primer día de valuación, tiempo $t_{0}$, la estrategia $\mathrm{BH}$ compra el activo y lo mantiene hasta que termine la ronda. El $A E$ ejecuta el AG para seleccionar la estrategia inicial y evalúa: comprar (Tiempo $t_{c}$ ); o mantenerse fuera (Tiempo $t_{v}$ ); conforme pasa el tiempo nuevos datos arriban y se ejecuta el AG, hasta que el autómata envía una señal de compra $t_{c}$; se compra el activo y se inicializa el cálculo del rendimiento de la estrategia del autómata $S_{0}=X_{c}$, al siguiente día se ejecuta nuevamente el AG y el autómata evalúa si se mantiene la posición de compra del activo o se vende; si se mantiene, se acumula el rendimiento generado en el nuevo día $S_{1}=S_{0}+$ $X_{c+1}$, se continua acumulando el rendimiento hasta que aparece la señal de venta (Tiempo $t_{v}$ ); se vende el activo y se calcula el rendimiento hasta ese día $S_{n+1}=X_{c}+X_{c+1}+\cdots+X_{v}$. El juego de compra-venta continúa conforme pasa el tiempo, a priori a las señales de compra o venta, en cada tiempo se ejecuta el AG que determina el cruce de medias móviles con las cuales se efectuará la compra o la venta, hasta que ocurra el tiempo de paro (Tiempo $t_{\tau}$ ). Esto es, si el tiempo transcurrido es mayor o igual a 65 días o el rendimiento acumulado $S_{n+1}$ rebasa algunos de los límites B o A se vende el activo; en la estrategia $\mathrm{BH}$ se vende también el activo y se calcula tanto el rendimiento del autómata $S_{n+1}$ como el rendimiento de la estrategia BH. Para ambos casos, una vez que se cumple el tiempo de paro se repite la prueba empezando el siguiente día en que terminó el ejercicio anterior, nuevamente se empieza con un rendimiento igual a cero, tanto en el rendimiento del autómata como de la estrategia BH. Esta serie de ejercicios o rondas se repiten siempre que la fecha no haya pasado del último día de prueba. Una vez se ha terminado el ejercicio, se suman los rendimientos obtenidos en cada ejercicio o ronda y se comparan para ver el desempeño del autómata versus la estrategia BH.

Descripción de los pasos:

Para cada instante de tiempo $t$ se ejecuta el AG para obtener la estrategia óptima.

1. Inicio en el tiempo $t_{0}$

a. $\quad$ Estrategia $\mathrm{BH}$, se compra el activo.

2. Tiempo $t_{c}$

a. $\quad$ Estrategia $\mathrm{BH}$, se mantiene la posición del activo.

b. $\quad A E$, se tiene la señal de comprar, se adquiere el activo. En caso de que se haya comprado el activo se mantiene la posición de compra.

3. Tiempo $t_{v}$

a. $\quad$ Estrategia $\mathrm{BH}$, se mantiene la posición del activo.

b. $\quad A E$, se tiene la señal de venta, se vende el activo. Se calcula el rendimiento acumulado. En caso de que no se haya adquirido el activo se mantiene la señal de no comprar.

4. Tiempo $t_{\tau}$.

a. Estrategia BH, se vende el activo. Se calcula el rendimiento acumulado. 
b. $\quad A E$, se vende el activo. Se calcula el rendimiento acumulado. En caso de que el $A E$ nunca haya comprado el activo, el rendimiento será cero.

5. Se repite el paso 1 , si aún no se ha llegado al tiempo de paro (Tiempo $t_{\tau}$ ) o se alcanzó alguno de los límites A o B.

\subsection{Parámetros del autómata y ejercicio}

Como parámetros para el autómata, se proponen como límites de ganancia: $A=12.4 \%, A=11.5 \%$, $A=7.7 \%$. Estos límites de ganancia se obtuvieron como la media de la tasa de rendimiento en cetes a 91 días, correspondientes a los tres intervalos de tiempo escogidos para la prueba6, más 2 veces la desviación estándar más 3\% por comisiones y costo de operación (2\% de comisiones por compra/venta7 y $1 \%$ como costo de operación ${ }^{8}$ ); se toman dos desviaciones estándar para tener una ganancia dos veces superior en volatilidad a la tasa libre de riesgos. Se propone un límite de pérdida $B=-3 \%$, del cual, su valor absoluto equivale a redondear el valor más bajo histórico, de la tasa de rendimiento de cetes a 91 días (la tasa de rendimiento cetes de $2.85 \%$ publicada el 4 de septiembre de 2014) y un tiempo de paro $\tau=65$ días el cual equivale a tres meses, similar al periodo de un pagaré de 91 días 9 . Los parámetros son propuestos por ser una ganancia a la tasa cetes en México, la selección óptima de esta tasa para diferentes perfiles de riesgo de inversionistas, escapan a este estudio.

El AG, así como las demás herramientas usadas se programaron mediante Matlab, se desarrolló una versión propia de AG con base al algoritmo propuesto por Goldberg (1987, pág. 61), el algoritmo propuesto se describe en la sección 3.3 de las metodologías. Se eligieron como parámetros: un periodo de prueba de 30 días para la función objetivo, una población inicial de 10 individuos, establecida como límite inferior; una tasa de mutación de 0.01\%, y 60 simulaciones para encontrar el cruce de medias móviles óptimo como lo propone Jiang y Szeto (2003).

\section{Resultados}

Se obtuvieron los rendimientos producidos por el $A E$ y los de la estrategia BH. Se calcularon tanto los rendimientos de forma individual para el IPC y DJ, como el de un portafolio con inversión del 50\% en cada uno de ellos en su composición. Los rendimientos se calcularon como la suma de los rendimientos ganados/perdidos para cada periodo de tiempo o ronda. Los tres escenarios de prueba

\footnotetext{
${ }^{6} \mathrm{El}$ límite $\mathrm{A}=12.4 \%$, corresponde a una muestra de tasas de rendimiento que abarcan el total del periodo de prueba, es decir, se tomó las tasas cetes a 91 días del 7 de abril de 2007 al 30 de septiembre de 2010; para el límite $\mathrm{A}=11.5 \%$, se tomó de muestra el periodo de crisis que abarca del 2 julio del 2007 al 21 abril de 2009 y para el límite $\mathrm{A}=7.7 \%$, el escenario de recuperación, se tomó la muestra que abarca del 17 de julio de 2009 al 30 de septiembre de 2010.

${ }^{7}$ De acuerdo al reporte de Finerio: https://blog.finerio.mx/blog/comisiones-clave-para-elegir-una-casa-de-bolsa. El $1 \%$ representa el valor máximo de comisión en México.

${ }^{8}$ Se considera cualquier efecto adverso temporal que sufre el inversor sobre el precio del activo al momento de la compra o venta.

${ }^{9}$ El tiempo de paro se calcula en días hábiles. El pagaré a 91 días toma en cuenta días naturales.
} 
fueron: total, del 2 de abril de 2007 al 30 de septiembre de 2010, éste abarca tanto el inicio de la crisis hipotecaria subprime, su terminación y recuperación; el segundo periodo, crisis, del 5 de julio de 2007 al 21 de abril de 2009, dura el tiempo en que los rendimientos de los índices estuvieron en descenso; y finalmente, el tercer periodo es el de recuperación o expansión de los mercados del 17 de julio de 2009 al 30 de septiembre de 2010.

Los rendimientos obtenidos provenientes del uso del $A E$ versus la estrategia BH para los tres escenarios se describen a continuación: En la Tabla 2, se muestran los rendimientos totales acumulados para escenario total; la Tabla 3 muestra el comportamiento del $A E$ versus la estrategia $\mathrm{BH}$ en el periodo que comprende a la crisis subprime, finalmente, la Tabla 3 muestra los resultados del $A E$ versus la estrategia $\mathrm{BH}$, durante el periodo de recuperación o expansión.

Tabla 2. Comparativo entre el rendimiento total obtenido por el $A E$ y el rendimiento obtenido por la estrategia BH, para el IPC, DJ y el portafolio de inversión, para el periodo del 2 de abril de 2007 al 30 de septiembre de 2010 (escenario total), variando el límite de ganancia A.

\begin{tabular}{|l|l|l|l|l|l|l|}
\hline & \multicolumn{2}{|l}{ Límite A=12.4\% } & \multicolumn{2}{l|}{ Límite A=11.5\% } & \multicolumn{2}{l|}{ Límite $\mathbf{A = 7 . 7 \%}$} \\
\hline \multirow{3}{*}{ Índice } & $\begin{array}{l}\text { Rendimiento } \\
\text { Acumulado } \\
\mathbf{A E}\end{array}$ & $\begin{array}{l}\text { Rendimiento } \\
\text { Acumulado } \\
\mathbf{B H}\end{array}$ & $\begin{array}{l}\text { Rendimiento } \\
\text { Acumulado } \\
\mathbf{A E}\end{array}$ & $\begin{array}{l}\text { Rendimiento } \\
\text { Acumulado } \\
\mathbf{B H}\end{array}$ & $\begin{array}{l}\text { Rendimiento } \\
\text { Acumulado } \\
\mathbf{A E}\end{array}$ & $\begin{array}{l}\text { Rendimiento } \\
\text { Acumulado } \\
\mathbf{B H}\end{array}$ \\
\hline IPC & $34.01 \%$ & $24.86 \%$ & $17.27 \%$ & $15.49 \%$ & $23.88 \%$ & $18.06 \%$ \\
\hline DJ & $-9.30 \%$ & $-11.52 \%$ & $-18.71 \%$ & $-20.03 \%$ & $-13.22 \%$ & $-13.53 \%$ \\
\hline Portafolio & $24.71 \%$ & $13.34 \%$ & $-1.44 \%$ & $-4.55 \%$ & $10.66 \%$ & $4.53 \%$ \\
\hline
\end{tabular}

Fuente: Elaboración propia.

En la Tabla 2 se observa que la ganancia acumulada que se obtuvo usando el $A E$ con el límite $\mathrm{A}=12.4 \%$ para el IPC fue de $34.01 \%$ versus el rendimiento de $24.86 \%$ de la estrategia BH. Para el caso del DJ, el $A E$ generó un rendimiento del $-9.30 \%$ versus un rendimiento acumulado $-11.52 \%$ de la estrategia $\mathrm{BH}$; si bien, para el DJ ambos rendimientos fueron negativos, el rendimiento obtenido por el autómata generó menor pérdida. Sumando los rendimientos como si se tuviera un portafolio, el $A E$ generó un rendimiento de $24.71 \%$ y la estrategia $\mathrm{BH} 13.34 \%$. Para el límite $\mathrm{A}=11.5 \%$, usando el $A E$, el IPC presentó una ganancia 1.78\% superior a la obtenida por la estrategia BH y el DJ una ganancia de $1.32 \%$ superior en comparación con lo generado por la estrategia $\mathrm{BH}$. Para el límite $\mathrm{A}=7.7 \%$, el $A E$ muestra una ganancia del portafolio del $10.66 \%$ versus el $4.53 \%$ usando la estrategia BH. Lo que prueba la ventaja de utilizar el $A E$, en un periodo de prueba en donde existió tanto una crisis como la recuperación del mercado.

Tabla 3. Comparativo entre el rendimiento total obtenido por el $A E$ y el rendimiento obtenido por la estrategia BH para el IPC, DJ y el portafolio de inversión, para el periodo del 2 de julio de 2007 al 21 de abril de 2009 (escenario crisis), variando el límite de ganancia A.

\begin{tabular}{|c|c|c|c|c|c|c|}
\hline & \multicolumn{2}{|c|}{ Límite $A=12.4 \%$} & \multicolumn{2}{|c|}{ Límite $A=11.5 \%$} & \multicolumn{2}{|l|}{ Límite $\mathrm{A}=7.7 \%$} \\
\hline Índice & $\begin{array}{l}\text { Rendimiento } \\
\text { Acumulado } \\
\text { AE }\end{array}$ & $\begin{array}{l}\text { Rendimiento } \\
\text { Acumulado } \\
\text { BH }\end{array}$ & $\begin{array}{l}\text { Rendimiento } \\
\text { Acumulado } \\
\text { AE }\end{array}$ & $\begin{array}{l}\text { Rendimiento } \\
\text { Acumulado } \\
\text { BH }\end{array}$ & $\begin{array}{l}\text { Rendimiento } \\
\text { Acumulado } \\
\text { AE }\end{array}$ & $\begin{array}{l}\text { Rendimiento } \\
\text { Acumulado } \\
\text { BH }\end{array}$ \\
\hline
\end{tabular}


REMEF (The Mexican Journal of Economics and Finance)

Autómata Evolutivo (AE) para el mercado accionario usando martingalas y un algoritmo genético

\begin{tabular}{|l|l|l|l|l|l|l|}
\hline IPC & $1.93 \%$ & $-44.32 \%$ & $-10.57 \%$ & $-43.56 \%$ & $3.92 \%$ & $-32.00 \%$ \\
\hline DJ & $-26.81 \%$ & $-86.27 \%$ & $-27.33 \%$ & $-57.17 \%$ & $-9.79 \%$ & $-53.54 \%$ \\
\hline Portafolio & $-24.88 \%$ & $-100.59 \%$ & $-37.89 \%$ & $-100.73 \%$ & $-5.87 \%$ & $-85.54 \%$ \\
\hline
\end{tabular}

Fuente: Elaboración propia.

En la Tabla 3, se muestra el comportamiento del $A E$ y la estrategia $\mathrm{BH}$ en el periodo que comprende a la crisis subprime. Para el límite $\mathrm{A}=12.4 \%$, se muestra como el $A E$ previno una pérdida importante, incluso generó una ganancia usando el $A E$ en el IPC, al tener un rendimiento de $1.93 \% \mathrm{y}$ de $-44.32 \%$ con la estrategia BH. Para el DJ, el $A E$ muestra una pérdida del $-26.81 \%$ versus $-86.27 \%$ del BH. Se observa que para este periodo se minimizó la pérdida, ya que, sumando los rendimientos del IPC y DJ obtenidos por el $A E$, se tiene un rendimiento total del $-24.88 \%$ versus el rendimiento usando la estrategia BH de $-100.59 \%$. Para el límite A=11.5\%, el $A E$ tuvo una pérdida en el IPC de $10.57 \%$ y la estrategia $\mathrm{BH}$ del $-43.56 \%$ y el índice DJ mostró una pérdida usando el $A E$ del $-27.33 \%$ y del $-57.17 \%$ para el $\mathrm{BH}$. De la misma forma, usando el límite A=7.7\% el $A E$ minimizó la pérdida al tener un rendimiento de portafolio del $-5.87 \%$ en comparación del BH que obtuvo el $-85.54 \%$.

Tabla 4. Comparativo entre el rendimiento total obtenido por el $A E$ y el rendimiento obtenido por la estrategia BH para el IPC, DJ, y el portafolio de inversión, para el periodo del 17 de julio de 2009 al 30 de septiembre de 2010 (escenario recuperación), variando el límite de ganancia A.

\begin{tabular}{|l|l|l|l|l|l|l|}
\hline & \multicolumn{2}{|l|}{ Límite A=12.4\% } & \multicolumn{2}{l|}{ Límite A=11.5\% } & \multicolumn{2}{l|}{ Límite A=7.7\% } \\
\hline \multirow{2}{*}{ Índice } & $\begin{array}{l}\text { Rendimiento } \\
\text { Acumulado } \\
\text { AE }\end{array}$ & $\begin{array}{l}\text { Rendimiento } \\
\text { Acumulado } \\
\text { BH }\end{array}$ & $\begin{array}{l}\text { Rendimiento } \\
\text { Acumulado } \\
\text { AE }\end{array}$ & $\begin{array}{l}\text { Rendimiento } \\
\text { Acumulado } \\
\text { BH }\end{array}$ & $\begin{array}{l}\text { Rendimiento } \\
\text { Acumulado } \\
\text { AE }\end{array}$ & $\begin{array}{l}\text { Rendimiento } \\
\text { Acumulado } \\
\text { BH }\end{array}$ \\
\hline IPC & $16.66 \%$ & $38.61 \%$ & $10.72 \%$ & $30.03 \%$ & $11.67 \%$ & $29.41 \%$ \\
\hline DJ & $7.56 \%$ & $25.78 \%$ & $5.48 \%$ & $21.64 \%$ & $-6.96 \%$ & $26.43 \%$ \\
\hline Portafolio & $24.22 \%$ & $64.39 \%$ & $16.20 \%$ & $51.66 \%$ & $4.70 \%$ & $55.83 \%$ \\
\hline
\end{tabular}

Fuente: Elaboración propia.

En la tabla 4 aparece el rendimiento obtenido para el $A E$ y la estrategia $\mathrm{BH}$ en el escenario de recuperación, se obtuvieron para ambos índices rendimientos positivos; con el límite A=12.4\% el $A E$ generó rendimientos del portafolio por $24.22 \%$ versus los rendimientos obtenidos por la estrategia BH que sumaron el $64.39 \%$; con el límite $\mathrm{A}=11.5 \%$, el $A E$ obtuvo un rendimiento del portafolio del $16.2 \%$ y la estrategia $\mathrm{BH}$ obtuvo del $51.66 \%$; usando el límite $\mathrm{A}=7.7 \%$, el $A E$ generó una ganancia del $4.7 \%$, mientras que la estrategia BH creó una ganancia de $55.83 \%$. En este escenario se observa como la estrategia $\mathrm{BH}$ fue superior al $A E$ sin importar el límite de ganancia.

Los resultados con diferentes límites de ganancia A observan el mismo patrón de comportamiento en relación a los rendimientos obtenidos del $A E$ versus la estrategia $\mathrm{BH}$, esto es, en el escenario total, el rendimiento del $A E$ fue superior al $\mathrm{BH}$, de la misma forma que en el escenario de crisis, finalmente en el escenario de recuperación, el rendimiento $\mathrm{BH}$ es superior al rendimiento del $A E$. Para el escenario total, el $A E$ con el límite $\mathrm{A}=12.4 \%$ obtuvo una ganancia acumulada del portafolio del $11.37 \%$, para el límite $A=11.5 \%$ la ganancia fue del $3.11 \%$ en términos absolutos y para el límite 
$\mathrm{A}=7.7 \%$ del $6.16 \%$ en comparación a la estrategia BH. Para el escenario de crisis, el $A E$ tuvo una ganancia del portafolio con el límite $\mathrm{A}=12.4 \%$ del $75.71 \%$ en términos absolutos; para $\mathrm{A}=11.5 \%$ del $62.84 \%$ y para $A=7.7 \%$ del $79.67 \%$ en comparación por usar la estrategia BH. En cambio, para el escenario de recuperación, el $A E$ generó un rendimiento del portafolio menor al de la estrategia $\mathrm{BH}$ por $40.17 \%$ para el límite $\mathrm{A}=12.4 \%$, un rendimiento del $35.46 \%$ menor a la estrategia $\mathrm{BH}$ para el límite $\mathrm{A}=11.5 \%$ y un rendimiento del $51.13 \%$ menor en términos absolutos para el límite $\mathrm{A}=7.7 \%$. En resumen, se observa que para el escenario total y el que abarca la crisis, en ambos índices el rendimiento generado por el $A E$ fue mejor al rendimiento de la estrategia $\mathrm{BH}$ y para el escenario de recuperación los rendimientos generados por la estrategia $\mathrm{BH}$ fueron superiores.

Se analiza el comportamiento del $A E$ en periodos de crisis y recuperación de manera independiente. Las siguientes gráficas muestran dos sub-periodos de prueba asociados: primero, al inicio y terminación de la crisis hipotecaria subprime (del 5 de julio de 2007 al 21 de abril de 2009); y segundo, la expansión o recuperación de los marcados (del 17 de julio de 2009 al 30 de septiembre de 2010), tanto para el IPC como el DJ. Se analiza únicamente lo correspondiente al límite A=12.4\% por ser el que busca mayor rendimiento. Las gráficas presentan, como bandas en tonalidad azul, los periodos de las posiciones de comprar y mantener del $A E$; y posiciones de venta o no invertir en bandas de tono gris; así también, las líneas color verde verticales marcan cuando se alcanzó el límite de ganancia $\mathrm{A}$, las líneas rojas señalan las fechas donde se alcanzaron el límite de pérdida $\mathrm{B}$ y finalmente las líneas negras señalan donde se ha cumplido el tiempo de permanencia del $A E$, es decir, el tiempo de paro tao.

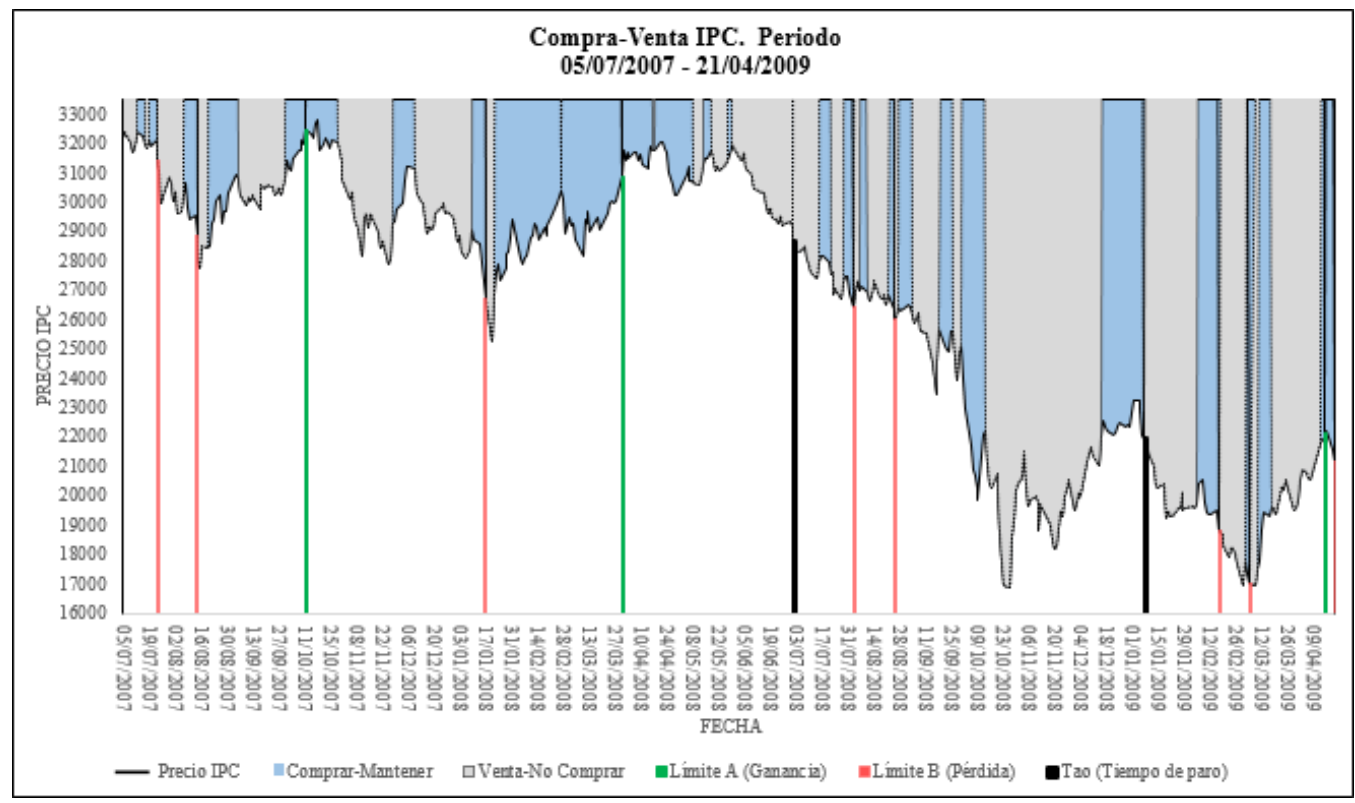

Gráfica 1. Precio de cierre del IPC y señales emitidas por el autómata del 5 de julio de 2007 al 21 de abril de 2009.

Fuente: Elaboración propia con datos de https://finance.yahoo.com/.

En la gráfica 1 se muestra gran parte del periodo de la crisis para el IPC del 5 de julio de 2007 al 21 de abril de 2009, se visualiza que en la mayoría de los casos cuando el índice bajó de precio se ejecutan señales de vender o no invertir y estas persisten durante los valles, una vez que el índice 
empieza a aumentar su valor le siguen las señales de compra y estas se mantienen hasta que ocurra un cambio de tendencia; en particular, se observan tres señales referentes al límite de ganancia A (líneas verdes), señales donde el rendimiento acumulado para el $\mathrm{AE}$ en la ronda fue superior al 12.4\%, siete señales de límite de pérdida B (líneas rojas); pérdida acumulada por el autómata mayor al -3\%; así mismo, se visualiza que en ese escenario se dieron dos tiempos de paro (línea negra), el cual se activó por estar en el "juego" 65 días y no rebasar algún límite de ganancia o pérdida.

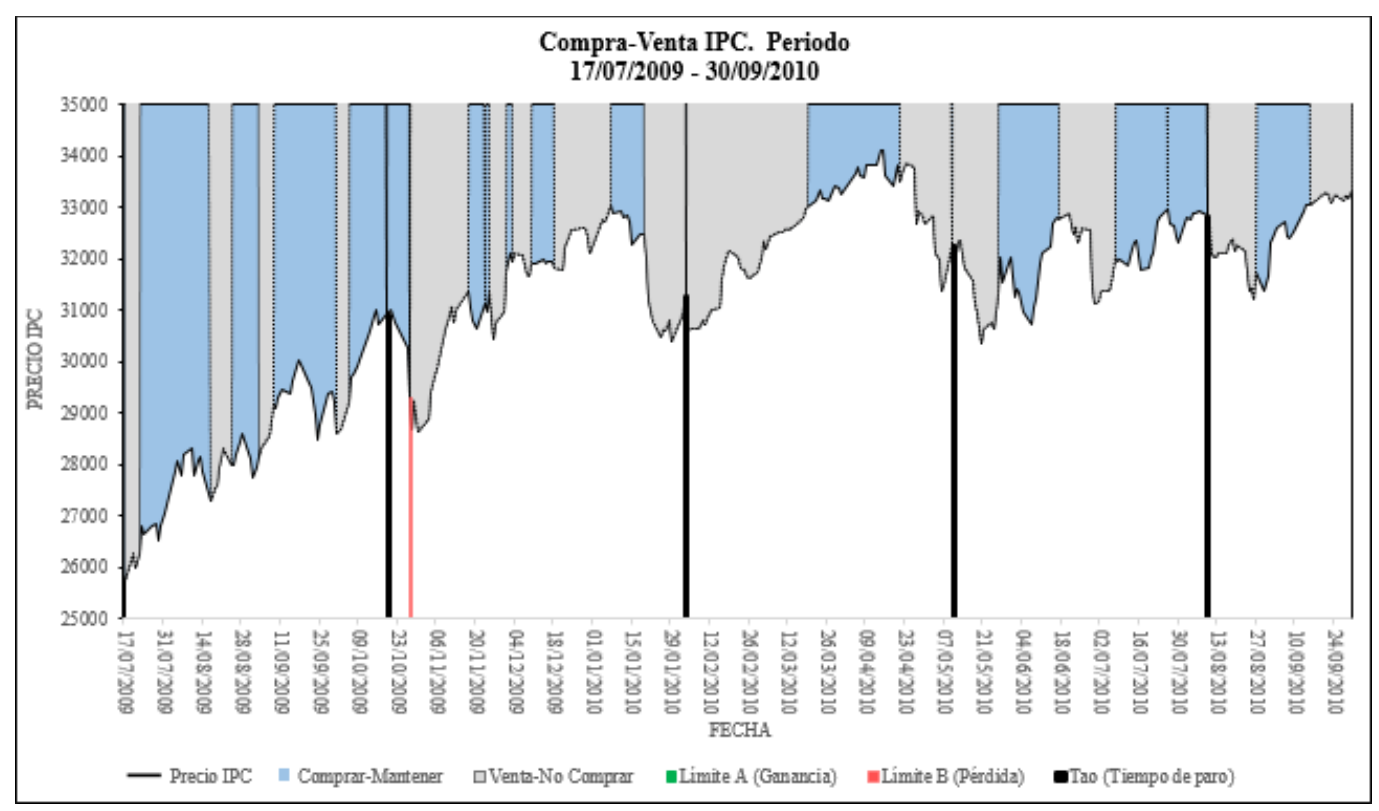

Gráfica 2. Precio de cierre del IPC y señales emitidas por el autómata del 17 julio de 2009 al 30 de septiembre de 2010.

Fuente: Elaboración propia con datos de https://finance.yahoo.com/.

La gráfica 2 presenta las posiciones que ejecutó el $A E$ en el periodo de recuperación del Índice de Precios y Cotizaciones (IPC), del 17 de julio de 2009 al 30 de septiembre de 2010. En este periodo se visualizan señales de comprar y mantener cuando aumenta el valor del precio para el índice, las señales se interrumpen pasando los picos por señales de venta y no invertir, esto ocurre en la mayoría del escenario salvo en dos intervalos: del 28 de octubre de 2009 al 17 de noviembre de 2009, donde se ejecuta un límite de pérdida B y del 8 de febrero de 2010 al 19 de marzo de 2010, donde para este último, la señal de compra se retrasa, dejando pasar una ganancia del 7.5\%, este retraso se debe a la volatilidad mostrada dos meses atrás y a la disminución en el precio del 7 de enero al 29 de enero de 2010, lo que hace que el AG tenga un retraso en la actualización de las señales. También se observa que se generan cuatro tiempos de paro, donde transcurridos más de 65 días hábiles se vende la posición y se obtiene el rendimiento acumulado para ese periodo.

En la Gráfica 3, se muestra el comportamiento del DJ y las posiciones del $A E$ en el periodo del 2 julio de 2007 al 21 de abril de 2009; se observa que la mayoría de las posiciones de venta o no invertir ocurren momentos antes de una disminución en el precio del índice, seguidas por intervalos donde se generan posiciones de compra, éstas posiciones son interrumpidas por límites de pérdida o tiempos de paro. Se muestra también que en este intervalo se generó un límite de ganancia A el 26 
de marzo de 2009 (se da el límite A al rebasar el 12.4\%), así mismo, se detonó el límite de pérdida B (al sobrepasar una pérdida del -3\%) en seis ocasiones. Durante este intervalo se generaron cuatro tiempos de paro debido a que 65 días antes no existió una ganancia superior al 12.4\% o una pérdida mayor al $-3 \%$.

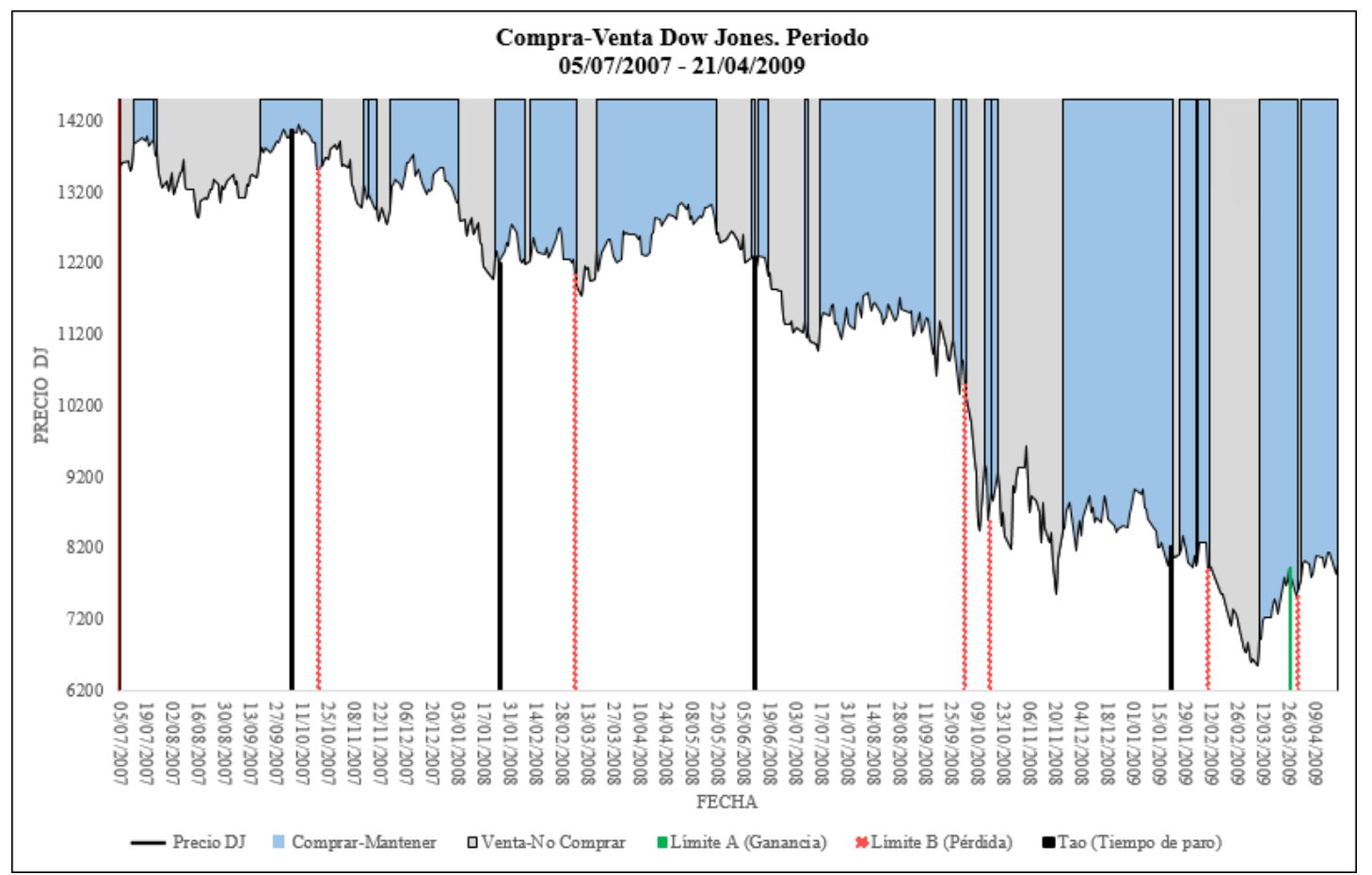

Gráfica 3. Precio de cierre del DJ y señales emitidas por el autómata del 5 julio de 2007 al 21 de abril de 2009.

Fuente: Elaboración propia con datos de https://finance.yahoo.com/.

La Gráfica 4 muestra el precio del DJ y las señales del autómata para un periodo marcado por una expansión en el mercado para ese índice, del 17 de julio de 2009 al 30 de septiembre de 2010. Se muestra como la mayoría de las señales de comprar o mantener son generadas después de un valle, al momento en que se incrementa el valor del índice, así mismo, la mayoría de las señales de venta o no invertir se generan cuando se disminuye el valor en el precio del índice, esto ocurre salvo en la primer y última señal de venta, la cual debido a la posición alcista que se llevaba, trae consigo un retraso al momento de ejecutar la señal; también que a pesar de ser un periodo alcista no hubo señales que marcaran un límite de ganancia A, esto debido a las fluctuaciones del índice. Así mismo, se ve como existen tres tiempos de paro los cuales indican que para estos intervalos no se sobrepasó algún límite de ganancia o pérdida; así mismo, que se detonaron dos límites de pérdida el 1 de junio de 2010 y el 29 del mismo mes y año. 


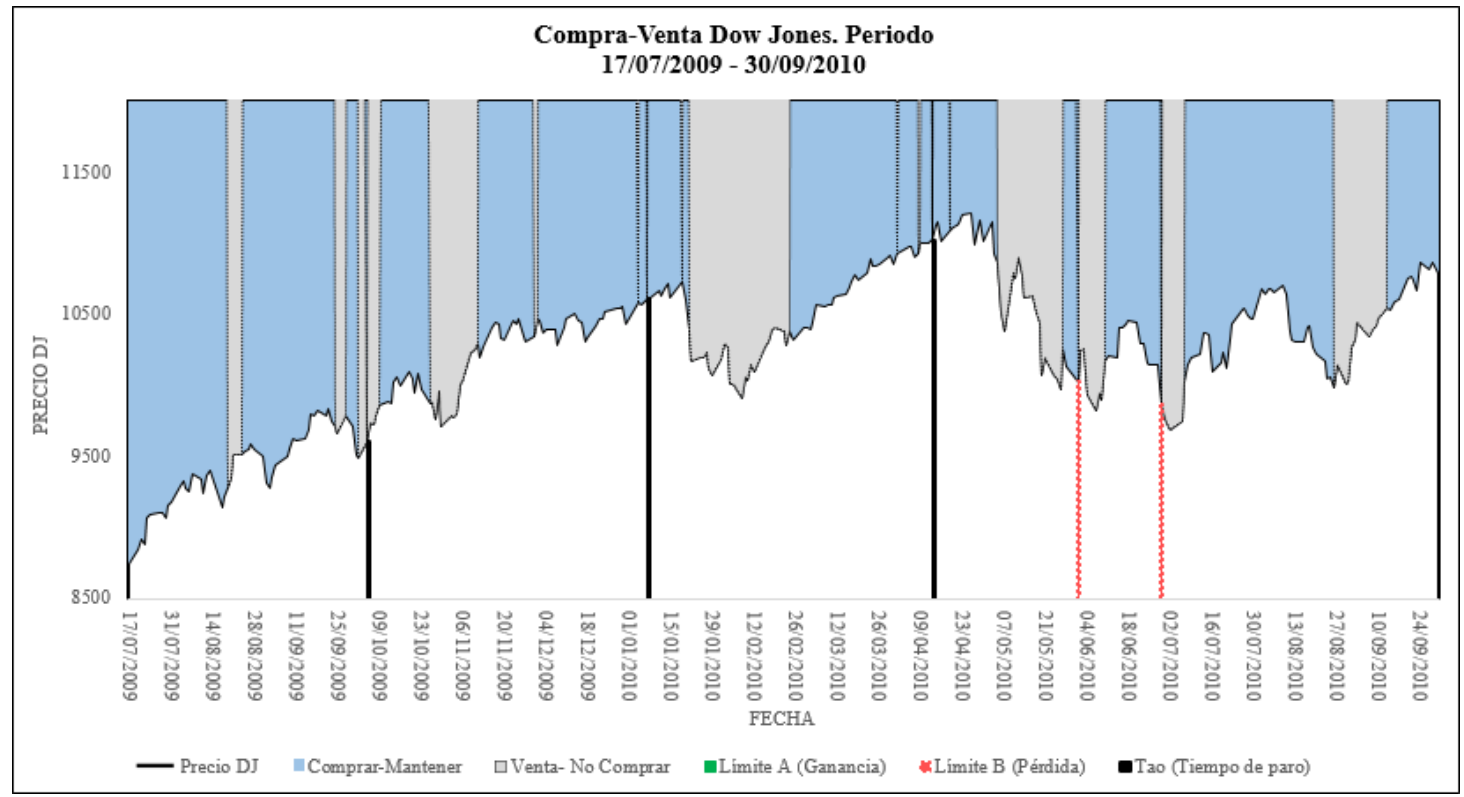

Gráfica 4. Precio de cierre del DJ y señales emitidas por el autómata del 17 julio de 2009 al 30 de septiembre de 2010.

Fuente: Elaboración propia con datos de https://finance.yahoo.com/.

\section{Conclusiones}

Con base a los resultados obtenidos, se tienen las siguientes conclusiones con relación a los tres diferentes escenarios del periodo que abarca del 2 de abril de 2007 al 30 de septiembre de 2010, para ambos índices, IPC, DJ y del portafolio de inversión; la estrategia $A E$ versus a la estrategia BH.

En el escenario de crisis, del 2 de julio de 2007 al 21 de abril de 2009, los rendimientos obtenidos fueron consistentes para los diferentes límites de ganancia propuestos $(A=12.4 \%$, $A=11.5 \%, A=7.7 \%)$. En particular, el rendimiento del $A E$ con límite $\mathrm{A}=12.4 \%$, con un portafolio de inversión $50 \%$ (IPC) y $50 \%$ (DJ) fue - $24.88 \%$ en comparación con el de la estrategia BH que sumó 100.59\%. De manera similar, para el límite $\mathrm{A}=11.5 \%$ y $\mathrm{A}=7.7 \%$, el $A E$ obtuvo un rendimiento de $37.89 \%$ y $-5.87 \%$, respectivamente; en comparación con $-100.73 \%$ y $-85.54 \%$, obtenidos de la estrategia BH. Es relevante mencionar que, para este escenario, la importancia de administrar el riesgo con el límite $\mathrm{B}$, minimizó la pérdida.

Con relación al periodo de expansión, que comprendió del 17 de julio de 2009 al 30 de septiembre de 2010, el $A E$ no tiene un mal desempeño; con el límite A=12.4\%, se obtiene un rendimiento del $16.66 \%$ para el IPC y del 7.56\% para el DJ. Sin embargo, la estrategia BH resultó ser la estrategia con mayor desempeño, al tener un rendimiento del 38.61\% para el IPC y del 25.78\% para el DJ. De manera similar, comparando los demás límites de ganancia, los rendimientos obtenidos por la estrategia $\mathrm{BH}$ fueron mejores a los obtenidos por el $A E$. Se concluye que la estrategia BH fue mejor en este periodo debido a que la tendencia alcista del periodo de prueba únicamente presentó pequeños intervalos con tendencia negativa, lo que provocó al $A E$ tomar posiciones de venta que resultaron en pérdidas de rendimiento (fue más costoso vender y volver a comprar). 
En el escenario total, que abarcó tanto la crisis como su recuperación, del 2 de abril de 2007 al 30 de septiembre de 2010; para el límite A=12.4\% el rendimiento total del portafolio de inversión usando el $A E$ brindó un rendimiento de $24.71 \%$, en comparación con la estrategia BH que fue del $13.34 \%$, es decir, el rendimiento usando el $A E$ fue $11.37 \%$ superior a la estrategia a BH. En el mismo sentido, usando los límites $\mathrm{A}=11.5 \%$ y $\mathrm{A}=7.7 \%$, el $A E$ obtuvo rendimientos superiores por $3.11 \% \mathrm{y}$ $6.13 \%$ respectivamente. Los resultados obtenidos fueron producto de los parámetros propuestos a través de la observación empírica explicada en la sección 3.1, sin embargo, la investigación podría fortalecerse con futuras aportaciones que puedan desarrollar métodos con base estadística para encontrar resultados óptimos en relación con el tiempo de permanencia, límites de ganancia y pérdida, así como el tamaño de las medias móviles.

En términos de la utilidad de la información histórica, el $A E$ demostró sus beneficios a través del uso de martingalas, se concluye que fue útil para anticiparse al comportamiento alcista o bajista de los índices. De igual forma, cuando se trató de optimizar las ganancias, fue adecuado establecer un límite de ganancia $\mathrm{A}$, tomando en cuenta la experiencia de la tasa libre de riesgo más un rendimiento razonablemente atractivo. También el uso de límites de pérdidas y permanencia en la inversión, minimizo la pérdida de capital al inversionista, en particular, el límite de pérdida $\mathrm{B}$, ayudó a minimizar pérdidas durante el periodo de crisis para ambos índices. Se concluye que el límite B y tiempo de permanencia son necesarios para delimitar el proceso de inversión de manera juiciosa.

Así también, se mostró que el AG ayudó a adaptar las estrategias conforme transcurrió el tiempo. Esto es de especial interés ya que no se pueden tener estrategias estáticas, ante un fenómeno como los movimientos del mercado accionario.

Por último, la aportación metodológica de este trabajo, parte de tomar el modelo de martingala para definir estrategias que van evolucionando conforme se mueven los mercados financieros, tomando en cuenta límites de administración de riesgo para minimizar las exposiciones de los riesgos a los que se está sujeto cualquier inversionista. En la metodología propuesta, se destaca la posibilidad de usar diferentes estrategias, mediante la definición de variables anticipadas; así también, su uso en combinación con procesos de optimización e inteligencia artificial es novedoso. Se propone para trabajos futuros, el uso de otras estrategias con base al estudio de ciclos de negocio, reconocimiento de patrones, componentes de frecuencia, entre otros; de la misma forma, probar otros métodos de inteligencia artificial o de aprendizaje de máquinas a fin de hacer que la estrategia aprenda más rápido y se ajuste mejor en el tiempo.

\section{Referencias}

[1] Allen, F., y Karjalainen, R. (1999). Using genetic algorithms to find technical trading rules. Journal of Financial Economics, 51 (2), 245-271. DOI: https://doi.org/10.1016/S0304-405X(98)00052-X

[2] Banerjee, S., y Breon-Drish, B. (2020). Strategic trading and unobservable information acquisition. Journal of Financial Economics, Article in Press. DOI: https://doi.org/10.1016/j.jfineco.2020.05.007.

[3] Brock, W., Lakonishok, J., y LeBaron, B. (1992). Simple Technical Trading Rules and the Stochastic Properties of Stock Returns. The Journal of Finance, 47 (5), 1731-1764. DOI: https://doi.org/10.1111/j.1540-6261.1992.tb04681.x. 
[4] Cavalcante, R., Brasileiro, R., Souza, V., Nobrega, J., y Oliveira, A. (2016). Computational Intelligence and Financial Markets: A Survey and Future Directions. Expert Systems with Applications, 55, 194-211. DOI: https://doi.org/10.1016/j.eswa.2016.02.006.

[5] Chan Phooi M'ng, J. (2018). Dynamically Adjustable Moving Avarage (AMA') technical analysis indicator to forecast Asian Tiger's futures markets. Physica A: Statistical Mechanics and its Applications, 509, 336 - 345. DOI: https://doi.org/10.1016/j.physa.2018.06.010

[6] Chen, Y., y Hao, Y. (2017). A feature weighted support vector machine and K-nearest neighbor algorithm for stock market indices prediction. Expert Systems with Applications, 80, 340-355. DOI: https://doi.org/10.1016/j.eswa.2017.02.044.

[7] Chiou, W., Knewtson, H., y Nofsinger, J. (2019). Paying attention to social media stocks. International Review of Economics \& Finance, 59, 106-119. DOI: https://doi.org/10.1016/j.iref.2018.08.009.

[8] Chung, H., y Shin, K. (2018). Genetic Algorithm-Optimized Long Short-Term Memory Network for Stock Market Prediction. Sustainability, 10 (10), 3765. DOI: https://doi.org/10.3390/su10103765.

[9] De la Uz, N. (2002). La hipótesis de Martingala en el mercado bursátil mexicano. Estudios Económicos: El Colegio de México, 17 (1), 91-127. https://www.jstor.org/stable/pdf/40311455.pdf?seq=1.

[10] Edwards, R., Magee, J., y Bassetti, W. (2018). Technical Analysis of Stock Trends. Boca Raton: CRC Press. DOI: https://doi.org/10.4324/9781315115719.

[11] Fama, E., y Blume, M. (1966). Filter Rules and Stock-Market Trading. The Journal of Business, 39 (1), 226-241. DOI: https://doi.org/10.1086/294849.

[12] Goldberg, D. (1989). Genetic Algorithms in Search, Optimization, and Machine Learning. The University of Alabama: Addison-Wesley Publishing Company, Inc. DOI: https://doi.org/10.5860/choice.27-0936.

[13] Golub, A., Glattfelder, J., y Olsen, R. (2017). The Alpha Engine: Designing an Automated Trading Algorithm. High Performance Computing in Finance, Chapman \& Hall/CRC Series in Mathematical Finance. DOI: https://doi.org/10.2139/ssrn.2951348.

[14] Gourieroux, C., y Jasiak, J. (2019). Robust analysis of the martingale hypothesis. Econometrics and Statistics, 9, 17-41. DOI: https://doi.org/10.1016/j.ecosta.2018.07.001.

[15] Groenewegen, L., y Hee, van K. (1976). Markov decisión processes and quasi-martingales. Memorandum COSOR: Technische Hogeschool Eindhoven., Vol. 7604. https://research.tue.nl/en/publications/markov-decision-processes-and-quasi-martingales-2.

[16] Grupo BMV. (2019). Muestra de emisoras vigentes que componen el S\&P/BMV Índice de Precios y Cotizaciones de la Bolsa Mexicana de Valores, S.A.B. de C.V. Ciudad de México, México: Grupo BMV. https://www.bmv.com.mx/es/emisoras/informacion-de-emisoras.

[17] Guo, Z., (2017). Order Flow and Exchange Rate Dynamics in Continuous Time: New Evidence from Martingale Regression. International Journal of Economics and Financial Issues, 7 (2), 507-512. DOI: http://dx.doi.org/10.2139/ssrn. 3013781.

[18] Harrison, J., y Kreps, D. (1979). Martingales and Arbitrage in Multiperiod Securities Markets. Journal of Economic Theory, 20 (3), 381-408. DOI: https://doi.org/10.1016/0022-0531(79)90043-7.

[19] Harrison, J., y Pliska, S. (1981). Martingales and stochastic integrals in the theory of continuous trading. Stochastic Processes and their Applications, 11 (3), 215-260. DOI: https://doi.org/10.1016/03044149(81)90026-0

[20] Holland, J. (1975). Adaptation in Natural and Artificial Systems. University of Michigan Press. https://mitpress.mit.edu/books/adaptation-natural-and-artificial-systems.

[21] Huang, B., Huan, Y., Da Xu, L., Zheng, L., y Zou, Z. (2019). Automated trading systems statistical and machine learning methods and hardware implementation: a survey. Enterprise Information Systems, 13 (1), 132-144. https://doi.org/10.1080/17517575.2018.1493145. 
[22] Huang, C., Hsu, C., Chen, C., y Chang, B. (2015). An Intelligent Model for Pairs Trading Using Genetic Algorithms. Computational Intelligence and Neuroscience. DOI: https://doi.org/10.1155/2015/939606.

[23] James, F. (1968). Monthly Moving Averages-An Effective Investment Tool? Journal of Financial and Quantitative Analysis, 3 (3), 315-326. DOI: https://doi.org/10.2307/2329816.

[24] Jiang, R., y Szeto, K. (2003). Extraction of investment strategies based on moving averages: A genetic algorithm approach. IEEE International Conference on Computational Intelligence for Financial Engineering, 2003.Proceedings, 403-410. DOI: https://doi.org/10.1109/cifer.2003.1196288.

[25] Lahmiri, S. (2018). A Technical Analysis Information Fusion Approach for Stock Price Analysis and Modeling. Fluctuation and Noise Letters, 17 (1). DOI: https://doi.org/10.1142/S0219477518500074.

[26] Lin, L., Cao, L., Wang, J., y Zhang, C. (2004). The Applications of Genetic Algorithms in Stock Market Data Mining Optimization. Management Information System, 10, 273-280. http://hdl.handle.net/10453/7024

[27] Lin, Y., Huang, C., y Tseng V. (2017). A novel methodology for stock investment using high utility episode mining and genetic algorithm. Applied Soft Computing, 59, 303-315. DOI: https://doi.org/10.1016/j.asoc.2017.05.032.

[28] Liu, X., An, H., Wang, L., y Jia, X. (2017). An integrated approach to optimize moving average rules in the EUA futures market based on particle swarm optimization and genetic algorithms. Applied Energy, 185. 1778-1787. DOI: https://doi.org/10.1016/j.apenergy.2016.01.045.

[29] Lobato Macedo, L., Godinho, P., y João Alves, M. (2020). A Comparative Study of Technical Trading Strategies Using a Genetic Algorithm. Computational Economics, 55 (1), 349-381. DOI: https://doi.org/10.1007/s10614-016-9641-9.

[30] Malmendier, U., Pouzo, D., y Vanasco, V. (2020). Investor experiences and financial market dynamics. Journal of Financial Economics, 136 (3), 597-622. DOI: https://doi.org/10.1016/j.jfineco.2019.11.002.

[31] Maymin, P. (2013). A New Kind of Finance. Irreducibility and Computational Equivalence, Part of the Emergence, Complexity and Computation book series, 2, 88-99. DOI: https://doi.org/10.1007/978-3642-35482-3_8.

[32] McCall, J. (2005). Genetic algorithms for modelling and optimization. Journal of Computational and Applied Mathematics, 184 (1), 205-222. DOI: https://doi.org/10.1016/j.cam.2004.07.034

[33] Mousinho Martins, T., y Ferreira Neves, R. (2020). Applying genetic algorithms with speciation for optimization of grid template pattern detection in financial markets. Expert Systems with Applications, 147. DOI: https://doi.org/10.1016/j.eswa.2020.113191

[34] Necchi, P. (2016). Reinforcement Learning for Automated Trading. Computer Science. http://www1.mate.polimi.it/ forma/Didattica/ProgettiPacs/BrambillaNecchi15-

16/PACS_Report_Pierpaolo_Necchi.pdf

[35] Päuna, C. (2018). Capital and Risk Management for Automated Trading Systems. Proceedings of the IE 2018 International Conference. DOI: 10.13140/RG.2.2.22827.90402

[36] Protter, P. (2015). Strict local martingales with jumps. Stochastic Processes and their Applications, 125 (4), 1352-1367. DOI: https://doi.org/10.1016/j.spa.2014.10.018

[37] Ray, A. (2019). Intelligent automation in capital markets: New tools for a smarter middle and back office. Celent Report. https://www.broadridge.com/_assets/pdf/broadridge-intelligent-automationbroadridge-excerpt.pdf

[38] Rincón, L. (2012). Introducción a los procesos estocásticos. Departamento de Matemáticas. Facultad de Ciencias. Universidad Nacional Autónoma de México. http://lya.fciencias.unam.mx/lars/libros/procesos2012.pdf

[39] Ritter, G. (2017). Machine Learning for Trading. Available at SSRN: https://doi.org/10.2139/ssrn.3015609 
[40] Román de la Sancha, L., Hernández Álvarez, F., y Rodríguez García, G. (2019). Co-movimientos entre los Índices Accionarios y Ciclos Económicos de Estados Unidos y México. Revista Mexicana de Economía y Finanzas Nueva Época, 14 (4). 693-714. DOI: https://doi.org/10.21919/remef.v14i4.352

[41] Ross, S. Westerfield R., Jaffe, J., Jordan, B. (2015). Corporate Finance. 11 ${ }^{\mathrm{TH}}$ Edition: McGrawHill. https://www.bookdepository.com/es/Corporate-Finance-Stephen-Ross/9780077861759

[42] Scarpati, C., Kroen, K., y Centrelli, C. (2018). Intelligent automation in capital markets operations. Pwc Financial Services. https://www.pwc.com/mx/es/publicaciones/c2g/2018-04-03-ia-capitalmarkets-operations.pdf?utm_source=RecursosAlumni\&utm_medium=InversionesYCrecimiento

[43] Schilling, L., y Uhlig H. (2019). Some simple bitcoin economics. Journal of Monetary Economics, 106, 16-26. DOI: https://doi.org/10.1016/j.jmoneco.2019.07.002

[44] Shynkevich, Y., McGinnity, T., Coleman, S., Belatreche A., y Li, Y. (2017). Forecasting price movements using technical indicators: Investigating the impact of varying input window length. Neurocomputing, 264, 71-88. DOI: https://doi.org/10.1016/j.neucom.2016.11.095

[45] Solimanpur, M., Mansourfar, G., y Ghayour, F. (2015). Optimum portfolio selection using a hybrid genetic algorithm and analytic hierarchy process. Studies in Economics and Finance, 32 (3), 379-394. DOI: https://doi.org/10.1108/SEF-08-2012-0085.

[46] Steele, J. (2001). Stochastic Calculus and Financial Applications. Springer-Verlag New York, DOI: https://doi.org/10.1007/978-1-4684-9305-4

[47] Tu, Z., y Lu, Y. (2004). A Robust Stochastic Genetic Algorithm (StGA) for Global Numerical Optimization. IEEE Transactions on Evolutionary Computation, 8 (5), 456-470. DOI: https://doi.org/10.1109/tevc.2004.831258

[48] Wang, L., An H., Liu X., y Huang, X., (2016). Selecting dynamic moving average trading rules in the crude oil futures market using a genetic approach. Applied Energy, 162, 1608-1618. DOI: https://doi.org/10.1016/j.apenergy.2015.08.132 